\title{
Global pathway view analysis of microRNA clusters in myasthenia gravis
}

\author{
CHUNRUI BO ${ }^{1 *}$, JIANJIAN WANG ${ }^{1 *}$, HUIXUE ZHANG $^{1 *}$, YUZE CAO $^{1,2}$, XIAOYU LU ${ }^{1}$, \\ TIANFENG WANG ${ }^{1}$, YU WANG ${ }^{1}$, SHUANG LI ${ }^{1}$, XIAOTONG KONG ${ }^{1}$, XUESONG SUN ${ }^{1}$, \\ ZHAOJUN LIU ${ }^{1}$, SHANGWEI NING ${ }^{3}$ and LIHUA WANG ${ }^{1}$ \\ ${ }^{1}$ Department of Neurology, The Second Affiliated Hospital, Harbin Medical University, Harbin, Heilongjiang 150081; \\ ${ }^{2}$ Department of Neurology, Peking Union Medical College Hospital, Peking Union Medical College and \\ Chinese Academy of Medical Sciences, Beijing 100730; ${ }^{3}$ College of Bioinformatics Science and Technology, \\ Harbin Medical University, Harbin, Heilongjiang 150081, P.R. China
}

Received June 19, 2018; Accepted October 25, 2018

DOI: $10.3892 / \mathrm{mmr} .2019 .9845$

\begin{abstract}
The significant roles of microRNAs (miRNAs) in the pathogenesis of myasthenia gravis (MG) have been observed in numerous previous studies. The impact of miRNA clusters on immunity has been demonstrated in previous years; however, the regulation of miRNA clusters in MG remains to be elucidated. In the present study, $245 \mathrm{MG}$ risk genes were collected and $99 \mathrm{MG}$ risk pathways enriched by these genes were identified. A catalog of $126 \mathrm{MG}$ risk miRNAs was then created; the MG risk miRNAs were located on each chromosome and a miRNA cluster was defined as a number of miRNAs with a relative distance of $<6 \mathrm{~kb}$ on the same sub-band, same band, same region and same chromosome. Furthermore, enrichment analyses were performed using the target genes of the MG risk miRNA clusters, and a number of risk pathways of each miRNA clusters were identified. As a result, 15 significant miRNA clusters associated with MG were identified. Additionally, the most significant pathways of the miRNA clusters were identified to be enriched on chromosomes 9, 19 and 22, characterized by immunity, infection and carcinoma, suggesting that the mechanism of MG may be associated with certain abnormalities of miRNA clusters on chromosomes 9, 19 and 22. The present study provides novel
\end{abstract}

Correspondence to: Professor Lihua Wang, Department of Neurology, The Second Affiliated Hospital, Harbin Medical University, 246 Xuefu Road, Harbin, Heilongjiang 150081, P.R. China

E-mail: wanglh211@163.com

Dr Shangwei Ning, College of Bioinformatics Science and Technology, Harbin Medical University, 157 Baojian Road, Harbin, Heilongjiang 150081, P.R. China

E-mail: ningsw@ems.hrbmu.edu.cn

*Contributed equally

Key words: myasthenia gravis, pathway analysis, microRNA clusters insight into a global pathway view of miRNA clusters in the pathogenesis of MG.

\section{Introduction}

Myasthenia gravis (MG) is an acquired neuromuscular autoimmune disease characterized by fluctuating muscle weakness and fatigue (1). The primary cause of MG is acetylcholine receptor antibodies, which destroy acetylcholine receptors on the posterior synaptic membrane of the neuromuscular junction with the involvement of cellular immunity and the complement system, so that it is not able to produce enough endplate potential, thereby causing the onset of the disease (2).

In previous years, a number of risk genes of MG have been identified, including circulating interleukin (IL)-17A, which was identified to be increased in patients with MG compared with normal controls, and increases of IL-17A were associated with general muscle weakness (3). Tumor necrosis factor- $\alpha$ (TNF- $\alpha$ ) is considered to be one of the most important cytokines in the pathogenesis of autoimmune MG, and the inhibition of TNF- $\alpha$ may have significant clinical efficacy for MG (4). Furthermore, chemokine CC motif receptor (CCR)9 and CCR7 have been demonstrated to be abnormally expressed at different thymocyte stages of $\mathrm{MG}$, and the overexpression of CCR9 and CCR7 in CD4-CD8-double negative thymocytes is associated with abnormal intrathymic T-cell differentiation in patients with MG (5). These observations suggest an increasing number of genes are crucial in the pathogenesis of MG. However, the majority of previous studies investigating MG risk genes have focused on only one or a few genes in cell lines without global analysis. Searches for a number of MG risk genes were performed in our previous studies $(6,7)$, however, the risk genes identified previously were not sufficiently comprehensive. The global pathway analysis of MG risk genes in the present study may assist in further characterizing the pathogenesis of MG.

Previous studies have demonstrated that microRNAs (miRNAs/miRs) are increasingly important in the pathogenesis of MG, and their aberrant expression may contribute towards the specific mechanism of MG. For example, miR-20b may 
inhibit nuclear factor of activated T cells (NFAT) signaling via the repression of NFAT5 and expression of calmodulin binding transcription activator 1 in thymoma-associated myasthenia gravis (TAMG) (8). The expression of the forkhead box (fox) p3 gene was modulated by miR-125a-5p, which is likely to be involved in the pathogenesis of TAMG (9). The abnormal expression of miR-15a facilitates pro-inflammatory cytokine production, at least in part by regulating the expression of C-X-C motif chemokine 10 (CXCL10), thereby contributing toward the immune response in MG (10). However, the potential mechanisms of miRNA in MG remain to be fully elucidated.

Certain previous studies have demonstrated that the pathogenesis of certain immune-associated diseases is associated with miRNA clusters on chromosomes. miRNAs are distributed across diverse genomic locations; although certain miRNAs are isolated, $\sim 50 \%$ are frequently physically clustered on the human genome to permit co-regulation, termed miRNA clusters (11). For example, the miR-106a-363 cluster encodes six miRNAs on the $\mathrm{X}$ chromosome, which include miR-18b, miR-106a and miR-363-3p, that were demonstrated to be involved in T-helper 17 cell differentiation, which further illustrates the association between the miR-106-363 cluster and immune-associated diseases $(12,13)$. The mirn23a gene is located on murine chromosome 8 and codes for three pre-miRNAs, miR-23a, miR-24-2 and miR-27a (14). The miR-23a-27a-24-2 miRNA cluster is an inhibitor of $\mathrm{B}$ cell development, and bone morphogenetic protein/mothers against decapentaplegic and Akt/FOXO1 signaling is critical for mirn23a-mediated immune cell regulation (14). However, there has been no overall investigation of miRNA clusters in MG. Furthermore, MG-associated miRNA clusters may have important regulated pathways that can clarify the mechanism of MG.

The present study identified the risk pathways regulated by MG risk genes and MG risk miRNAs. Subsequently, each MG risk miRNA on each chromosome was located and 15 significant miRNA clusters associated with MG were identified. The risk pathways of each of the 15 miRNA clusters were identified, which can further assist in elucidating the potential mechanism of miRNA clusters in MG at the post-transcriptional regulation level.

\section{Materials and methods}

Human MG risk gene data. Human MG risk gene data was acquired using the following two approaches: i) Gene information was obtained by searching certain current databases, including DisGeNET (http://www.disgenet. org/web/DisGeNET/menu) (15) and Online Mendelian Inheritance in Man (http://www.omim.org/) (16); and ii) using the protocols published in our previous studies $(6,7)$, the gene was notably differentially expressed in more than five MG samples using dependable biological laboratorial techniques, and 9,514 items were browsed by manually collecting literature using the terms [myasthenia gravis (MeSH Terms) and English (Language)] and the species 'Homo sapiens' published prior to March 1st, 2017 on the PubMed database (https://www.ncbi. nlm.nih.gov/pubmed); the eligible genes were selected. An update was made to the previous catalog of $162 \mathrm{MG}$ risk genes described in our previous study (7) to 245 .
Human MG risk miRNA data, miRNA location data and miRNA target data. Human miRNA information was acquired from miRBase (http://www.mirbase.org/) (17) and the miRNA location information was obtained from NCBI-GENE database (https://www.ncbi.nlm.nih.gov/gene/) (18). MG risk miRNAs were additionally gathered in two ways. To begin with, literature published prior to March 1st, 2017 was manually searched using the protocols described in our previous study (7); the keywords 'miRNA' and 'myasthenia gravis' or 'microRNA' and 'myasthenia gravis' or 'miR' and 'myasthenia gravis' were searched in PubMed. Additionally, MG risk miRNAs were downloaded from The Nervous System Disease NcRNAome Atlas database (http://www.bio-bigdata.net/nsdna/) (19). Validated human miRNA target data was obtained from the miRTarBase (http://mirtarbase.mbc.nctu.edu.tw/php/index.php) (20).

Functional enrichment analysis of MG risk genes. Pathway data were obtained from the Kyoto Encyclopedia of Genes and Genomes (KEGG) database (https://www.genome. $\mathrm{jp} / \mathrm{kegg} /$ ) (21) to dissect numerous specific MG risk pathways. In order to identify the MG risk pathways in which MG risk genes were enriched, KEGG pathway enrichment analysis was conducted by applying functional annotation tools in the Database for Annotation, Visualization, and Integrated Discovery (DAVID) (https://david.ncifcrf.gov/) (22). The significance level of KEGG pathway enrichment was calculated using a cut-off of $\mathrm{P}<0.05$. Gene Ontology (GO) annotation (23) was additionally performed using DAVID for the MG risk gene catalog. A GO term was considered significantly enriched if it had a false discovery rate (FDR) value $<0.05$.

Calculation of miRNA distances on chromosomes and definition of miRNA clusters. The location information of all acquired MG risk miRNAs on chromosomes were obtained using the NCBI-GENE database (https://www.ncbi.nlm.nih. gov/gene/) (18) to obtain the detailed location of each miRNA, in addition to their starting and ending points. Subsequently, neighboring miRNAs on the same chromosome, same long arm or short arm, same region, same band and even the same sub-band were selected, and the distances between these adjacent miRNAs were calculated. Therefore, a miRNA cluster was defined as a number of miRNAs with a relative distance of $<6 \mathrm{~kb}$ on the same sub-band, same band, same region and same chromosome.

Functional enrichment analysis of MG risk miRNA clusters. Enrichment analyses were performed using DAVID with the target genes of miRNAs on each MG risk miRNA cluster that had been defined. The significance level of KEGG pathway enrichment was calculated using a cut-off of $\mathrm{P}<0.05$. However, the GO enrichment was considered to be significant using a cut-off of FDR $<0.01$.

\section{Results}

Update of the MG risk gene catalog and identification of key risk pathways of human $M G$ risk genes. In our previous study (7), a catalog of $162 \mathrm{MG}$ risk genes was created and 45 MG risk pathways were identified $(\mathrm{P}<0.05)$. Using our previous study, the catalog of MG risk genes was updated and a new 

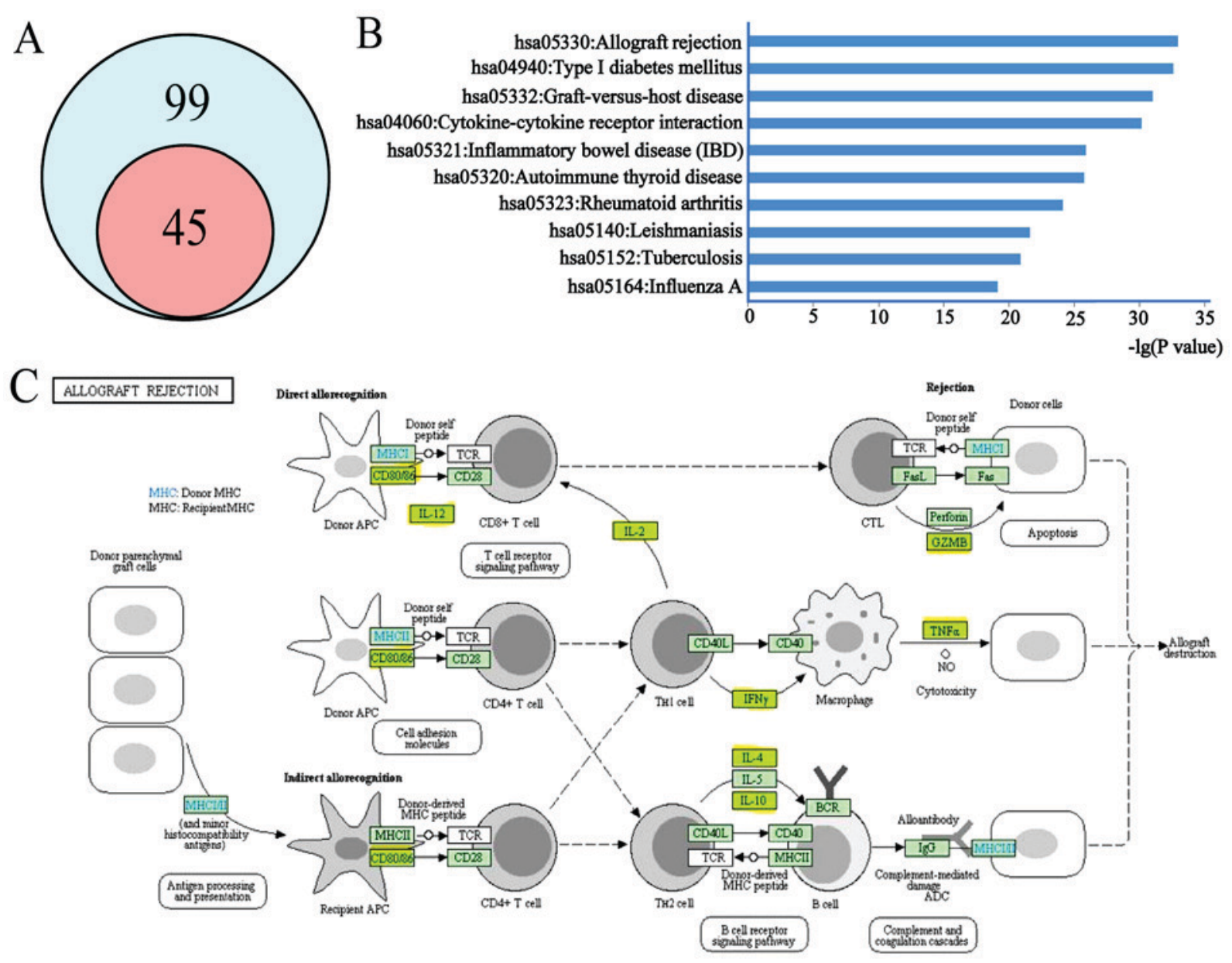

Figure 1. Risk pathways of human MG. (A) Wayne diagram of the comparison of pathways in two studies. Blue represents the $99 \mathrm{MG}$ risk pathways $(\mathrm{P}<0.05$ ) identified in the present study; red represents the $45 \mathrm{MG}$ risk pathways identified in our previous study [Ref (7)]. (B) Top 10 MG risk pathways of MG risk genes with Kyoto Encyclopedia of Genes and Genomes enrichment $(\mathrm{P}<0.05)$. (C) Dissection of the top MG risk pathway (hsa05330: Allograft rejection). Proteins coded by MG risk genes are indicated with a yellow background. MG, myasthenia gravis.

catalog of $245 \mathrm{MG}$ risk genes, which had been confirmed by the experiments, was created. Of all 245 genes, 131 risk genes were collected manually from the literature and 114 risk genes were downloaded from public databases. In view of these MG risk genes, 99 MG risk pathways $(\mathrm{P}<0.05)$ were identified using KEGG enrichment analysis in DAVID. The same P-value $(\mathrm{P}<0.05)$ was selected as that used in the previous study (7). Compared with the previous study (7), 83 additional MG risk genes were excavated and 44 additional MG risk pathways were newly identified. A Wayne diagram was constructed to clarify the association between pathways that were identified in the present study and our previous study. As presented in Fig. 1A, it was demonstrated that the pathways that were enriched in our previous study were all included in the 99 MG risk pathways identified in the present study. This was considered an update to our previous findings.

The novel identified top 10 significantly enriched pathways in the present study are presented in Fig. 1B. It was demonstrated that five of the top 10 pathways were associated with 'human disease: Immune disease' in the KEGG database, further illustrating the association between MG and autoimmunity. It was additionally identified that hsa05330 (allograft rejection) was the most significantly enriched pathway through KEGG enrichment analysis. A total of eight MG risk genes were involved in this pathway and important in $\mathrm{MG}$, as presented in Fig. 1C.

In addition, functional enrichment analysis of these MG risk genes was performed and $119 \mathrm{GO}$ analysis entries (FDR $<0.05$ ) were obtained, including 92 biological processes (BPs), 11 molecular functions (MFs) and 16 cellular components (CCs). The top three significant BPs were immune response, inflammatory response and T cell co-stimulation, further illustrating the fundamental characteristics of immunity on MG.

Construction of the MG risk miRNA dataset and its distribution on chromosomes. A catalog of 126 miRNAs was constructed and the positions of each MG risk miRNA on 23 chromosomes were located by searching information on the mirBase database (17) and NCBI-GENE database (18). The distribution of these 126 MG risk miRNAs in 23 pairs of chromosomes is presented in Table I. The distribution of MG risk miRNAs on 23 chromosomes was plotted according to the chromosome distribution and the position of each miRNA on the chromosomes, as presented in Fig. 2. It was demonstrated that the majority of miRNAs are distributed in the $\mathrm{X}$ chromosome, and chromosomes 17, 19, 1, 3, 7, 9 and 22 , and the number of miRNAs on the $\mathrm{X}$ chromosome was the largest, with 16 miRNAs on this chromosome. However, 
Table I. Distribution of 126 MG risk miRNAs on 23 chromosomes.

\begin{tabular}{|c|c|}
\hline Chromosome & MG risk miRNAs \\
\hline 1 & $\begin{array}{l}\text { hsa-miR-320b-1, hsa-miR-197-3p, hsa-miR-30e-5p, hsa-miR-34a-5p, hsa-miR-92b-3p, hsa-miR-199a-3p, } \\
\text { hsa-miR-181b-5p, hsa-miR-29b-3p, hsa-miR-29c-3p, hsa-miR-664a-5p, hsa-miR-320b-2 }\end{array}$ \\
\hline 2 & hsa-miR-933, hsa-miR-375, hsa-miR-149-5p \\
\hline 3 & $\begin{array}{l}\text { hsa-let-7g-5p, hsa-miR-191-3p, hsa-miR-564, hsa-miR-425-5p, hsa-miR-26a-5p, hsa-miR-563, } \\
\text { hsa-miR-885-5p, hsa-miR-15b-5p, hsa-miR-16-5p }\end{array}$ \\
\hline 4 & hsa-miR-574-3p \\
\hline 5 & hsa-miR-584-5p, hsa-miR-145-5p, hsa-miR-146a-5p \\
\hline 6 & hsa-miR-30a-5p, hsa-miR-548a-3p \\
\hline 7 & $\begin{array}{l}\text { hsa-miR-25-3p, hsa-miR-93-5p, hsa-miR-106b-5p, hsa-miR-106b-3p, hsa-miR-129-1-3p, hsa-miR-183-5p, } \\
\text { hsa-miR-29a-3p, hsa-miR-29b-3p }\end{array}$ \\
\hline 8 & hsa-miR-486-5p, hsa-miR-320a, hsa-miR-548a-3p, hsa-miR-1234-3p \\
\hline 9 & $\begin{array}{l}\text { hsa-let-7a-5p, hsa-let-7f-5p, hsa-let-7f-1-3p, hsa-let-7d-5p, hsa-miR-24-3p, hsa-miR-181a-2-3p, } \\
\text { hsa-miR-181b-5p, hsa-miR-602 }\end{array}$ \\
\hline 10 & hsa-miR-107 \\
\hline 11 & $\begin{array}{l}\text { hsa-miR-129-2-3p, hsa-miR-210-3p, hsa-miR-1237-3p, hsa-miR-192-5p, hsa-miR-139-5p, hsa-miR-125b-5p, } \\
\text { hsa-let-7a-5p }\end{array}$ \\
\hline 12 & hsa-miR-200c-3p, hsa-miR-1228-3p, hsa-miR-26a-5p, hsa-let-7i-5p, hsa-miR-548c-3p, hsa-miR-331-3p \\
\hline 13 & $\begin{array}{l}\text { hsa-miR-320d, hsa-miR-16-5p, hsa-miR-15a-5p, hsa-miR-17-5p, hsa-miR-20a-5p, hsa-miR-92a-3p, } \\
\text { hsa-miR-1267 }\end{array}$ \\
\hline 14 & hsa-miR-1260a, hsa-miR-342-3p, hsa-miR-345-5p, hsa-miR-665, hsa-miR-494-3p \\
\hline 15 & hsa-miR-629-5p \\
\hline 16 & hsa-miR-365a-3p, hsa-miR-1225-5p, hsa-miR-940, hsa-miR-140-5p, hsa-miR-140-3p \\
\hline 17 & $\begin{array}{l}\text { hsa-miR-33b-3p, hsa-miR-324-3p, hsa-miR-22-5p, hsa-miR-144-3p, hsa-miR-144-5p, hsa-miR-193a-3p, } \\
\text { hsa-miR-193a-5p, hsa-miR-423-3p, hsa-miR-451a, hsa-miR-10a-5p, hsa-miR-142-3p, hsa-miR-142-5p, } \\
\text { hsa-miR-21-5p, hsa-miR-634, hsa-miR-338-3p }\end{array}$ \\
\hline 18 & hsa-miR-1539, hsa-miR-122-5p \\
\hline 19 & $\begin{array}{l}\text { hsa-miR-24-3p, hsa-miR-27a-3p, hsa-miR-23a-3p, hsa-miR-181c-5p, hsa-miR-1470, hsa-miR-1238-3p, } \\
\text { hsa-miR-199a-3p, hsa-miR-150-5p, hsa-let-7e-5p, hsa-miR-125a-3p, hsa-miR-125a-5p, hsa-miR-523-3p, } \\
\text { hsa-miR-520d-5p, hsa-miR-518d-3p }\end{array}$ \\
\hline 20 & hsa-miR-1825, hsa-miR-296-5p \\
\hline 21 & hsa-let-7c-5p, hsa-miR-125b-5p, hsa-miR-155-5p \\
\hline 22 & $\begin{array}{l}\text { hsa-miR-185-5p, hsa-miR-130b-3p, hsa-miR-1281, hsa-miR-1249-3p, hsa-let-7a-3p, hsa-let-7a-5p, } \\
\text { hsa-let-7b-5p, hsa-let-7b-3p }\end{array}$ \\
\hline $\mathrm{X}$ & $\begin{array}{l}\text { hsa-let-7f-5p, hsa-miR-188-5p, hsa-miR-362-5p, hsa-miR-500a-3p, hsa-miR-532-5p, hsa-miR-221-3p, } \\
\text { hsa-miR-421, hsa-miR-652-3p, hsa-miR-766-3p, hsa-miR-18b-3p, hsa-miR-20b-5p, hsa-miR-363-3p, } \\
\text { hsa-miR-92a-3p, hsa-miR-424-5p, hsa-miR-320d, hsa-miR-505-5p }\end{array}$ \\
\hline
\end{tabular}

MG, myasthenia gravis; miRNA/miR, microRNA.

no MG risk miRNAs were identified to be distributed on the Y chromosome, suggesting that the incidence of MG-related diseases is higher in females compared with males. This result is consistent with the majority of the reported proportions of males to females in immunological disease distribution (24-26).

Furthermore, neighboring miRNAs, which are on the same chromosome, same long arm or short arm, same region, same band and same sub-band were selected, and the distances between these adjacent miRNAs were calculated. Subsequently, a miRNA cluster was defined as a number of adjacent miRNAs with a relative distance of $<6 \mathrm{~kb}$. As a result,
15 miRNA clusters were identified; these are listed in Table II and these clusters are marked in red in Fig. 2.

KEGG enrichment analysis of each MG risk miRNA cluster on 23 chromosomes. Human MG risk miRNA target genes were obtained from miRTarBase (20). Enrichment analyses were subsequently performed using the target genes of each miRNA contained in 15 MG risk miRNA clusters on each chromosome. For example, there are two clusters on chromosome 3: Cluster miR-191-3p-425-5p and cluster miR-16-5p-15b-5p. Subsequently, KEGG enrichment analysis with the target genes of the four MG risk miRNAs contained in miRNA 

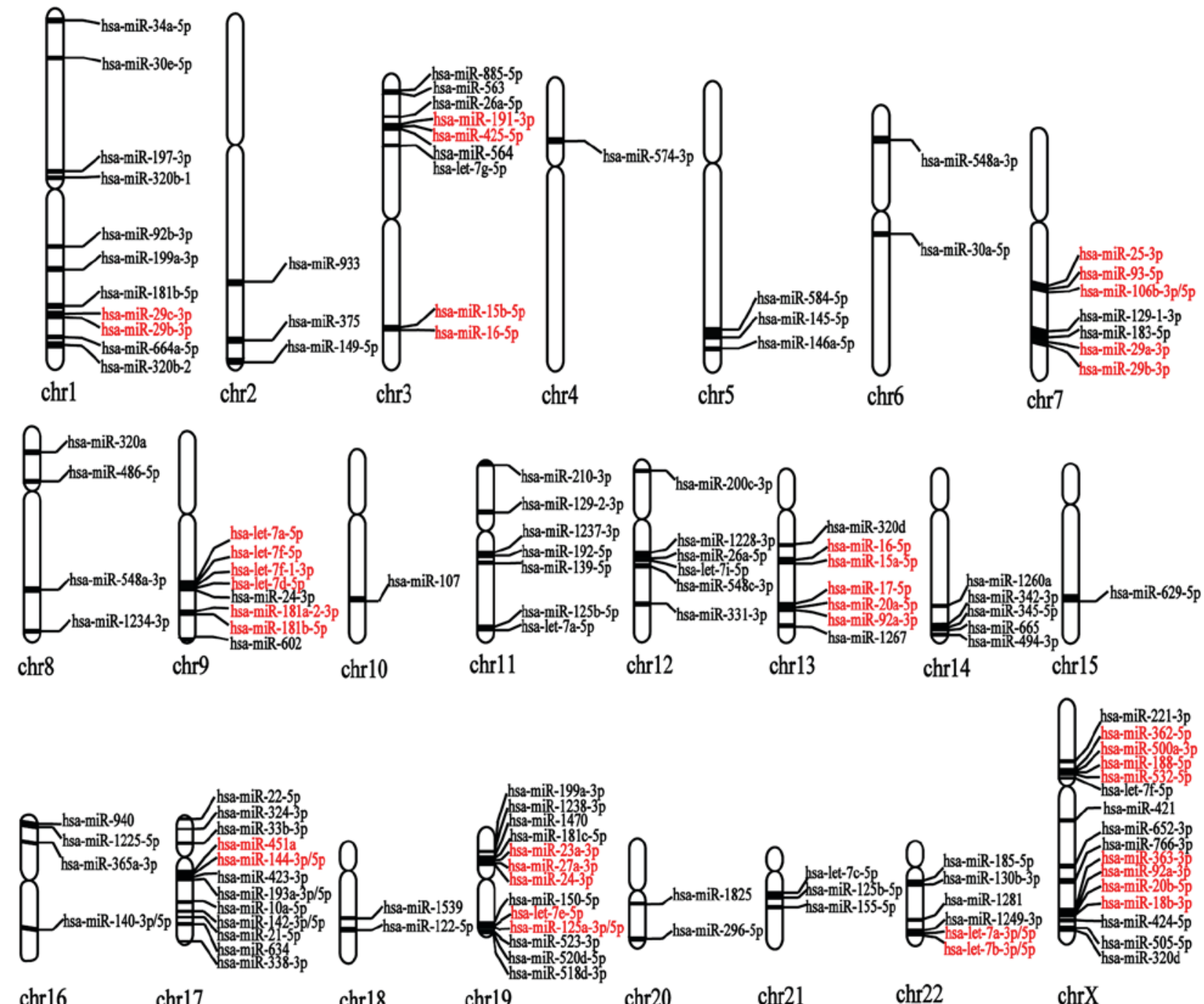

(hen

chr14

chr15

$\begin{array}{llll}\operatorname{chr16} & \operatorname{chr17} & \operatorname{chr} 18 & \operatorname{chr} 19\end{array}$

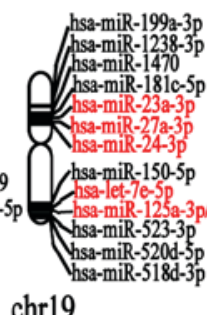

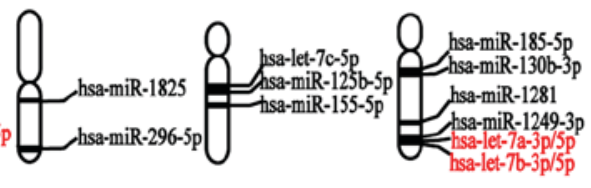

$\operatorname{chr} 20$

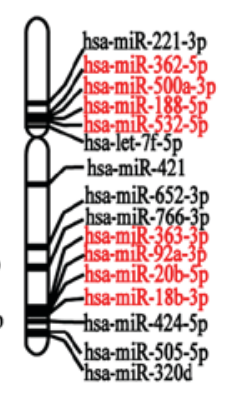

$\operatorname{chr} \mathrm{X}$

Figure 2. Distribution of MG risk miRNAs on 23 chromosomes. The miRNA clusters are indicated in red. MG, myasthenia gravis; miRNA/miR, microRNA; chr, chromosome.

clusters was performed. A cut-off of $\mathrm{P}<0.05$ was considered to indicate a statistically significant difference. Therefore, numerous significant pathways on chromosome 3 were identified, and a classification of the enriched pathways was made through KEGG maps. According to the aforementioned steps, the remaining clusters underwent the same enrichment analysis. The results of the KEGG enrichment analysis $(\mathrm{P}<0.05)$ are presented in Fig. 3.

In addition, the meaningful pathways $(\mathrm{P}<0.05)$ enriched by MG risk miRNA clusters and the 99 risk pathways $(\mathrm{P}<0.05)$ enriched by MG risk genes in the aforementioned results were intersected, and the pathways marked in red when enriched in the two sets of results. The majority of the identified pathways belonged to the following categories: 'Environmental information processing-signal transduction'; 'Human disease-cancer'; 'Human diseases-infectious diseases'; 'Organismal systems-endocrine system'; and 'Organismal systems-immune system' in the KEGG database; this suggests that, in addition to the immune system, cancerous pathways, infectious pathways and endocrine pathways can also be used to regulate miRNA clusters and thus functionally characterize MG. The results additionally suggested that the most significant pathways are enriched on chromosomes 9,19 and 22, indicating that the mechanism of MG may be associated with certain abnormalities of chromosomes 9,19 and 22.

Pathway hsa05210 (colorectal cancer): A pathway regulated by the majority of MG risk miRNA clusters. A transverse comparison of the pathways of KEGG enrichment analysis $(\mathrm{P}<0.05)$ was subsequently made using the target genes of each MG risk miRNA cluster. The results demonstrated that pathway hsa05210 (colorectal cancer) was regulated by the majority of miRNA clusters (eight miRNA clusters, including 22 miRNAs). hsa05210 (colorectal cancer) was additionally a significant pathway that was enriched by the aforementioned MG risk genes. The miRNA clusters that regulated the hsa05210 (colorectal cancer) pathway are identified in Table III. The hsa05120 (colorectal cancer) pathway is presented in Fig. 4. The target genes of these eight miRNA clusters are marked in yellow, and the miRNAs that regulate the target genes are marked in red, additionally presented in Fig. 4.

Through analysis, among all the target genes of the eight miRNA clusters that regulated the hsa05120 pathway, transforming growth factor $\beta 1$ (TGFB1), caspase 3 (CASP3), 
Table II. Details of the 15 miRNA clusters identified with a distance of $<6 \mathrm{~kb}$ on genomes.

\begin{tabular}{ll}
\hline Chromosome & \multicolumn{1}{c}{ miRNAs within each cluster } \\
\hline 1 & hsa-miR-29b-3p, hsa-miR-29c-3p \\
3 & hsa-miR-191-3p, hsa-miR-425-5p \\
3 & hsa-miR-16-5p, hsa-miR-15b-5p \\
7 & hsa-miR-106b-3p, hsa-miR-106b-5p, \\
& hsa-miR-93-5p, hsa-miR-25-3p \\
7 & hsa-miR-29b-3p, hsa-miR-29a-3p \\
9 & hsa-let-7d-5p, hsa-let-7f-5p,hsa-let-7f-1-3p, \\
& hsa-let-7a-5p \\
9 & hsa-miR-181b-5p, hsa-miR-181a-2-3p \\
13 & hsa-miR-15a-5p, hsa-miR-16-5p \\
13 & hsa-miR-92a-3p, hsa-miR-20a-5p, \\
& hsa-miR-17-5p \\
17 & hsa-miR-144-3p, hsa-miR-144-5p, \\
& hsa-miR-451a \\
19 & hsa-miR-23a-3p, hsa-miR-27a-3p, \\
& hsa-miR-24-3p \\
& hsa-miR-125a-3p, hsa-miR-125a-5p, \\
& hsa-let-7e-5p \\
& hsa-let-7b-3p, hsa-let-7b-5p, hsa-let-7a-3p, \\
& hsa-let-7a-5p \\
& hsa-miR-532-5p, hsa-miR-500a-3p, \\
& hsa-miR-362-5p, hsa-miR-188-5p \\
& hsa-miR-92a-3p, hsa-miR-363-3p, \\
& hsa-miR-20b-5p, hsa-miR-18b-3p \\
&
\end{tabular}

miRNA/miR, microRNA.

KRAS, MYC and B-cell lymphoma 2 (BCL2), were MG risk genes. TGFB1 (27,28), CASP3 (29), KRAS (30), MYC (31) and BCL2 (32) have all been demonstrated to be associated with miRNA clusters. Overall, hsa05210 (colorectal cancer) may be a key pathway connecting miRNA clusters with MG.

KEGG enrichment analysis of two MG risk miRNA clusters on the X chromosome. As the X chromosome was identified to have the largest number of MG risk miRNAs and is significant in autoimmune diseases, the two miRNA clusters on the X chromosome: Hsa-miR-532-5p, hsa-miR-500a-3p, hsa-miR-362-5p, hsa-miR-188-5p and hsa-miR-92a-3p, hsa-miR-363-3p, hsa-miR-20b-5p, hsa-miR-18b-3p, were analyzed. A separate KEGG enrichment analysis $(\mathrm{P}<0.05)$ was performed for the target genes of these two miRNA clusters on the $\mathrm{X}$ chromosome; as a result, 36 pathways were significantly enriched $(\mathrm{P}<0.05)$. Classification of these pathways was performed through the KEGG database. The results demonstrated that eight pathways were associated with 'Environmental information processing-signal transduction', nine were associated with 'Human diseases-cancers', four were associated with 'Human diseases-infectious diseases', four were associated with 'Organismal systems-endocrine system', two were associated with 'Organismal systems-immune system', and two
Table III. miRNA clusters regulating the hsa05210 (colorectal cancer) pathway.

\begin{tabular}{ll}
\hline Chromosome & \multicolumn{1}{c}{ miRNA clusters } \\
\hline 3 & hsa-miR-16-5p, hsa-miR-15b-5p \\
9 & hsa-let-7d-5p, hsa-let-7f-5p, hsa-let-7f-1-3p, \\
& hsa-let-7a-5p \\
& hsa-miR-15a-5p, hsa-miR-16-5p \\
13 & hsa-miR-92a-3p, hsa-miR-20a-5p, \\
13 & hsa-miR-17-5p \\
& hsa-miR-144-3p, hsa-miR-144-5p, \\
17 & hsa-miR-451a \\
& hsa-miR-125a-3p, hsa-miR-125a-5p, \\
& hsa-let-7e-5p \\
& hsa-let-7b-3p, hsa-let-7b-5p, hsa-let-7a-3p, \\
22 & hsa-let-7a-5p \\
& hsa-miR-92a-3p, hsa-miR-363-3p, \\
hsa-miR-20b-5p, hsa-miR-18b-3p
\end{tabular}

miRNA/miR, microRNA.

were associated with 'Organismal systems nervous system', as presented in Fig. 5. These results were consistent with the results described for the KEGG enrichment analysis of target genes of miRNA clusters on 23 chromosomes.

GO enrichment analysis of each MG risk miRNA cluster on 23 chromosomes. In addition to KEGG analysis, functional enrichment analysis using the target genes of $15 \mathrm{MG}$ risk miRNA clusters on each chromosome was performed, and a total of 74 GO_BP terms were obtained (FDR <0.01). However, with the exception of chromosomes 9, 17, 19 and 22, no enriched pathways were identified on the other chromosomes. Subsequently, these entries were classified and divided into the following categories: 'Apoptotic process', 'cell adhesion', 'cell cycle', 'cell differentiation', 'cell migration', 'cell proliferation', 'cellular response', 'material phosphorylation', 'physiological response', 'reproduction and expression of genetic material', 'signal transduction' and 'tissue development'. These results are summarized in Fig. 6. The results of GO_BP enrichment were additionally concentrated on chromosomes 9, 19 and 22 . Combined with the results of KEGG enrichment analysis, these results indicated that the pathogenesis of MG may be closely associated with chromosomes 9, 19 and 22 .

\section{Discussion}

MG is a neuromuscular autoimmune disease; however, the specific mechanism of MG remains to be fully elucidated. The analysis of MG risk miRNA clusters and their regulation of relevant pathways may assist in elucidating their potential involvement in the pathogenesis of MG. However, at present, there are few studies on miRNA clusters and pathways of MG. In the present study, global analysis of MG-associated miRNA clusters and their potential mechanism was performed based on the enrichment analysis of MG risk genes and miRNAs. 

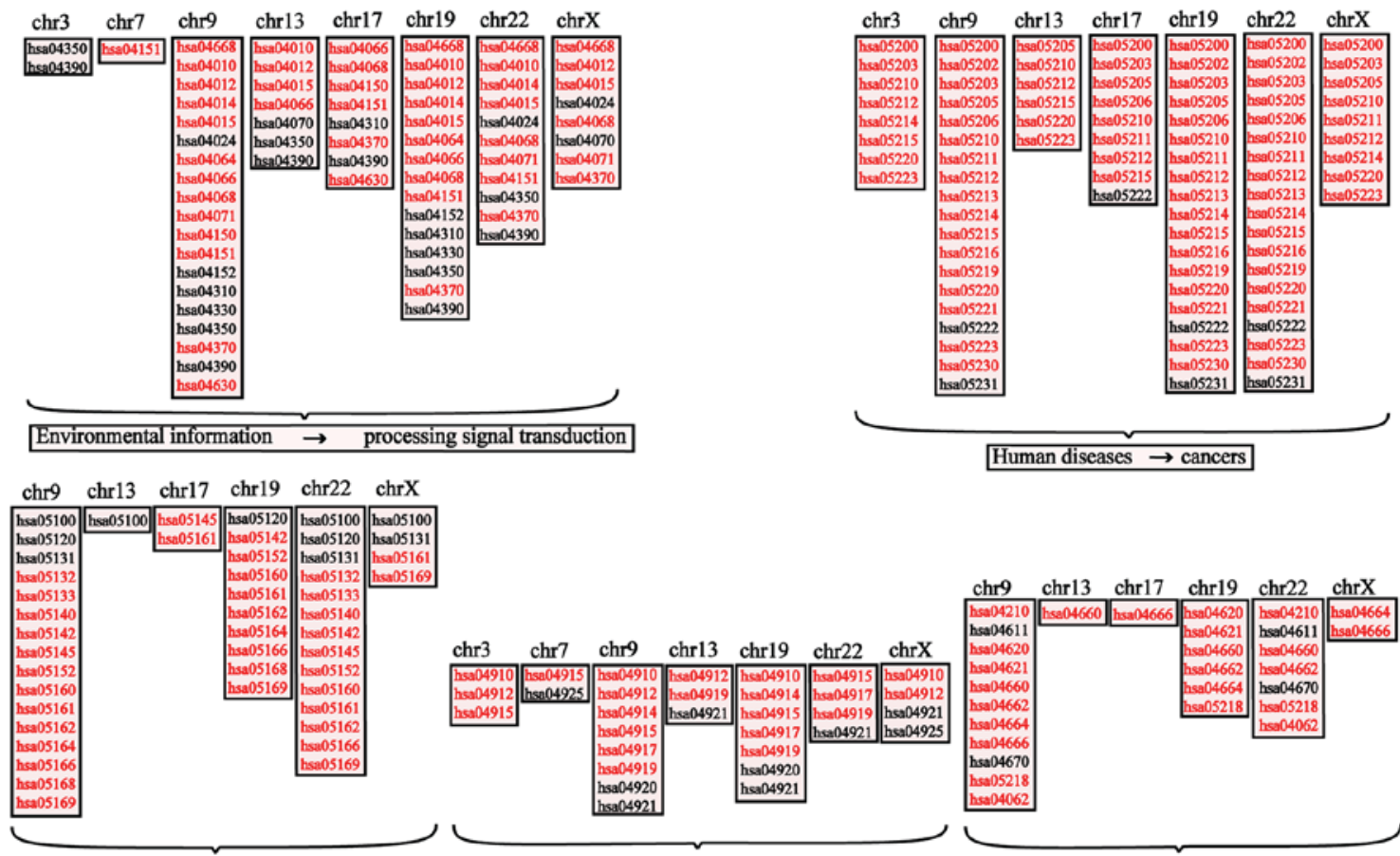

Human diseases $\rightarrow$ infectious diseases

Organismal systems $\rightarrow$ endocrine system

Organismal systems $\rightarrow$ immune system

Figure 3. Kyoto Encyclopedia of Genes and Genomes enrichment analysis with the target genes of $15 \mathrm{MG}$ risk miRNA clusters on each chromosome. Red represents the intersections between the meaningful pathways $(\mathrm{P}<0.05)$ enriched by MG risk miRNA clusters and the 99 risk pathways $(\mathrm{P}<0.05)$ enriched by MG risk genes. MG, myasthenia gravis; miRNA, microRNA; chr, chromosome.

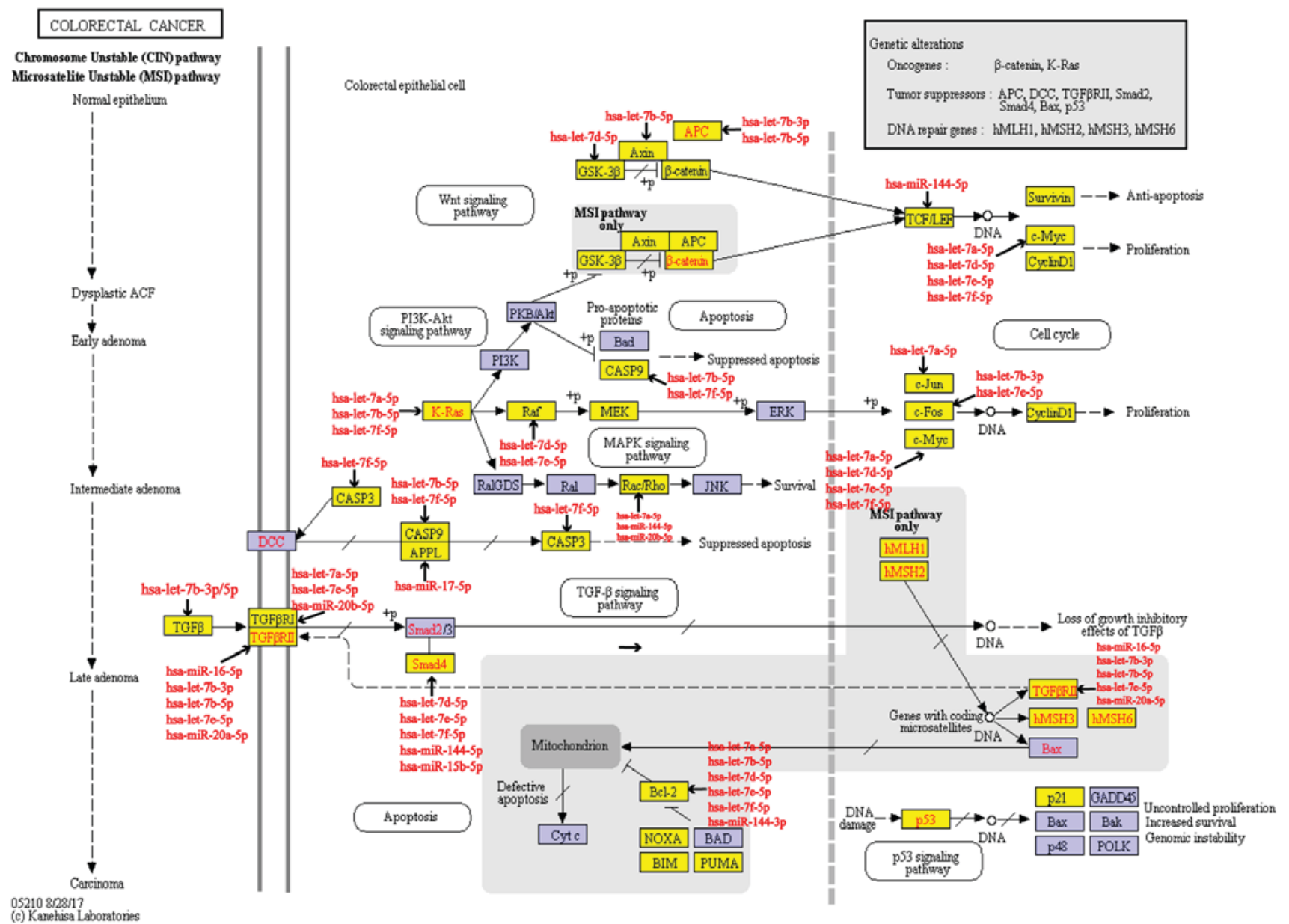

Figure 4. Dissection of pathway hsa05120 (colorectal cancer). Red represents the miRNA clusters regulating the pathway, yellow represents the target genes of these miRNAs. miRNA/miR, microRNA. 


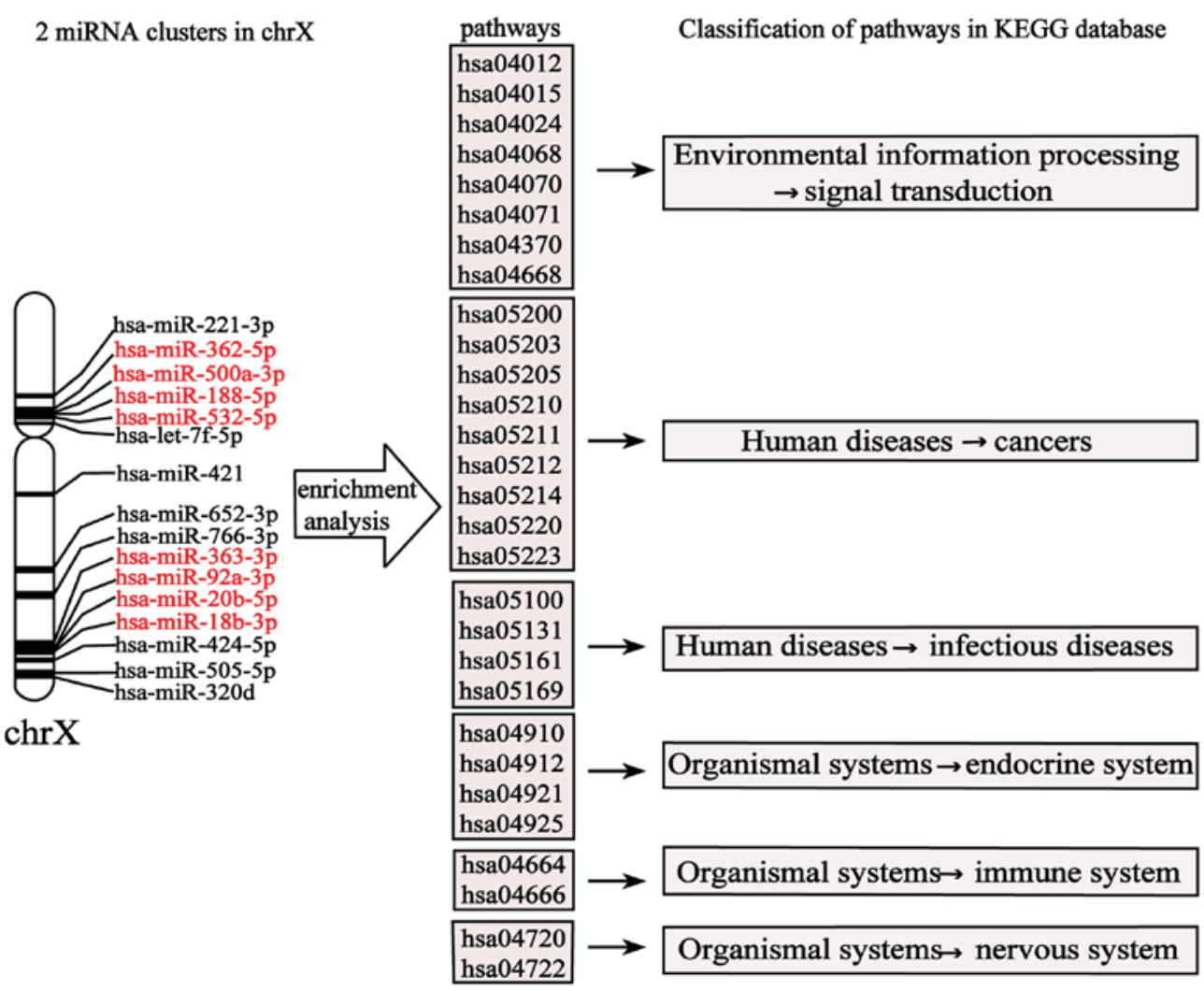

Figure 5. KEGG enrichment analysis of target genes of two MG risk miRNA clusters on the $\mathrm{X}$ chromosome $(\mathrm{P}<0.05)$. miRNAs in red represent the two miRNA clusters identified in the present study. The middle column shows pathways identified by target genes of the two miRNA clusters through KEGG enrichment analysis $(\mathrm{P}<0.05)$. The column on the right shows the classification of pathways in the KEGG database. MG, myasthenia gravis; miRNA/miR, microRNA; chrX, X chromosome; KEGG, Kyoto Encyclopedia of Genes and Genomes.
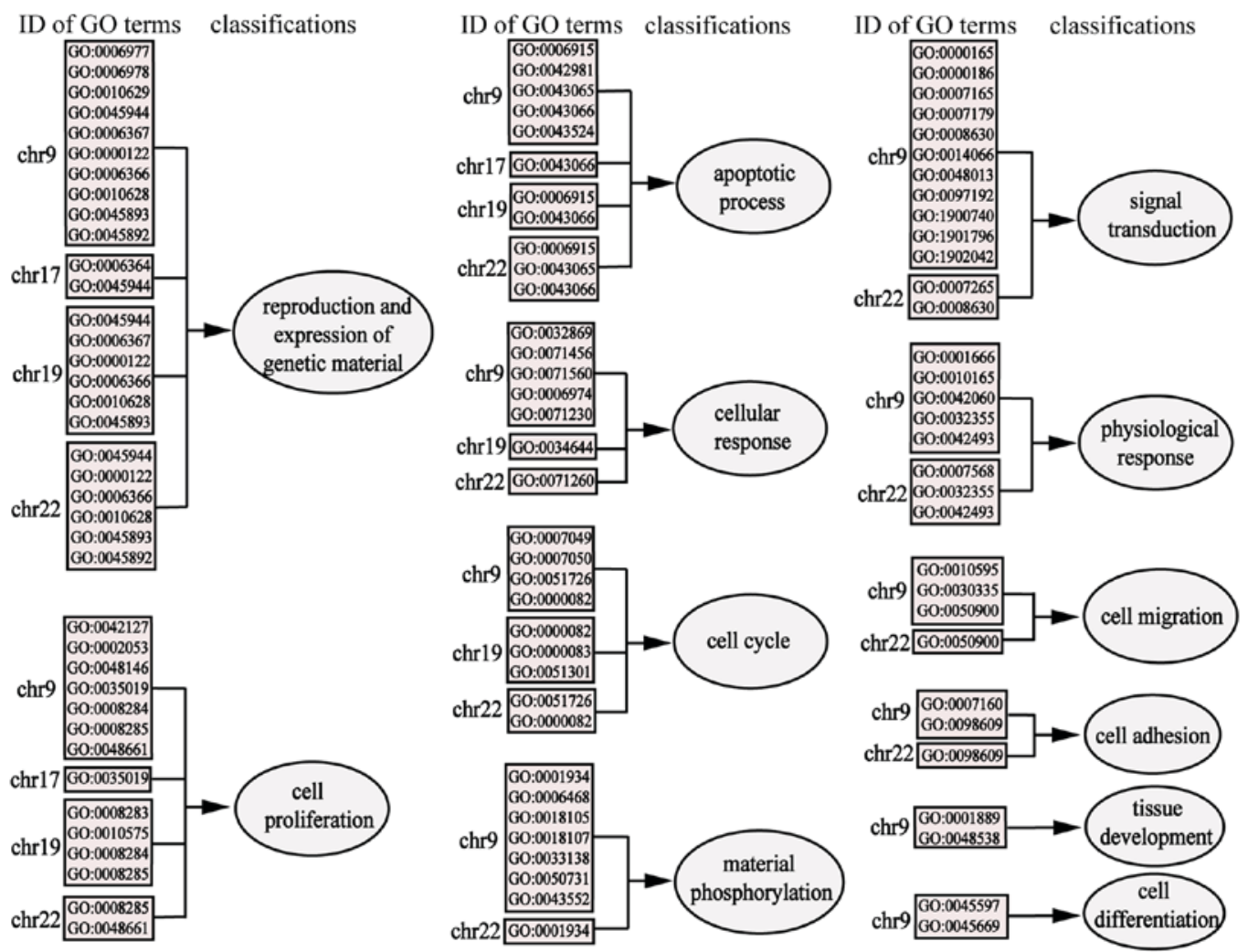

Figure 6. GO_BP functional enrichment analysis (FDR<0.01) of the target genes of 15 MG risk miRNA clusters on each chromosome. The results were centered on chromosome 9, 17, 19 and 22. The significant GO_BP terms (FDR<0.01) were divided into 12 classifications, which are shown within ellipses. The ID numbers of the GO_BP terms are within different rectangles according to the chromosome and classification. GO, Gene Ontology; BP, biological process; MG, myasthenia gravis; miRNA, microRNA; chr, chromosome; FDR, false discovery rate. 
MG risk genes were collected and the MG risk pathways enriched by these genes were identified. Among the top 10 pathways enriched by the MG risk genes, it was identified that the majority of these pathways were associated with immunity when searching the KEGG database. The MG risk miRNAs were subsequently obtained and each of the $126 \mathrm{MG}$ risk miRNAs from each chromosome were located; 15 miRNA clusters were identified to be significantly associated with MG. In addition, enrichment analysis was performed using the target genes of the $15 \mathrm{MG}$ risk miRNA clusters with a distance of $<6 \mathrm{~kb}$ on each chromosome. Furthermore, a number of risk pathways of each of the 15 miRNA clusters were identified and the most significant pathways were demonstrated to be enriched on chromosomes 9, 19 and 22. This suggests that the mechanism of MG may be associated with certain abnormalities of chromosomes 9, 19 and 22.

The top 10 pathways identified using the data of $\mathrm{MG}$ risk genes provides an overview of the pathogenesis of MG. Among the top $10 \mathrm{MG}$ risk pathways, five were associated with immunity and three were revealed to be associated with 'Human diseases: Infectious diseases', suggesting that microorganism infection may be involved in the pathogenesis of MG, providing novel insight into MG. For example, an active Epstein-Barr virus (EBV) infection in the thymus of patients with MG has been reported previously, suggesting that EBV may contribute towards the onset or maintenance of the autoimmune response by targeting toll-like receptor (TLR)7 and TLR9 in the intrathymic pathogenesis of MG (33). Through pathway analysis, 99 significant pathways were identified that characterized MG in different aspects. The most significant pathway identified was hsa05330 (allograft rejection). MG has been reported as a rare complication of chronic graft-vs.-host disease (GVHD) following allogeneic hematopoietic stem cell transplantation in several case reports (34-36), which is consistent with the results of the present study in which hsa05330 (allograft rejection) had the most significant association with MG.

miRNA clusters are a novel concept and indicates that miRNAs are clustered on the human genome to permit co-regulation. miRNA clusters have the ability to regulate more biological pathways than normal miRNAs, as one cluster often contains several miRNAs that have the same biological function. The present study located each MG risk miRNA on each chromosome and identified 15 significant miRNA clusters, with distances $<6 \mathrm{~kb}$, associated with MG. Among the 15 miRNA clusters, at least five miRNA clusters were identified that have been reported to be associated with immunity, including the miR-25-93-106b cluster (37), which comprises hsa-miR-106b-3p, hsa-miR-106b-5p, hsa-miR-93-5p and hsa-miR-25-3p on chromosome 7; the let-7 family cluster (38), comprising hsa-let-7d-5p, hsa-let-7f-5p, hsa-let-7f-1-3p and hsa-let-7a-5p on chromosome 9; the miR-17-92 cluster (39-42), comprising hsa-miR-92a-3p, hsa-miR-20a-5p and hsa-miR-17-5p on chromosome 13; the miR-23a-27a-24-2 cluster (14,43-45), comprising hsa-miR-23a-3p, hsa-miR-27a-3p and hsa-miR-24-3p on chromosome 19; and the miR-106a-363 cluster (12,13), comprising hsa-miR-92a-3p, hsa-miR-363-3p, hsa-miR-20b-5p and hsa-miR-18b-3p on the X chromosome.

The present study identified numerous examples associated with miRNA clusters that may regulate the immune system.
The miR-25-93-106b cluster has been demonstrated to regulate tumor metastasis and immune evasion via the modulation of CXCL12 and programmed death ligand 1 (37). The miR-17-92 cluster is critical in early B cell development and $\mathrm{T}$ cell differentiation $(46,47)$, and its functions involve autoimmune disorders, including GVHD and leukemia (39), experimental autoimmune encephalomyelitis (41), chronic infections (48) and cancer (49). The mirn23a gene, which encodes miR-23a, -24-2 and -27a, transforms into the mirn23a cluster and is also central to $\mathrm{T}$ cell differentiation (44); however, antagonizes B cell development (45) unlike the miR-17-92 cluster. The mirn23a cluster was previously reported to be involved in the pathogenesis of immune-associated diseases, including hematopoiesis (14), CKD-induced muscle atrophy (50), leukemia (51) and a number of types of carcinoma (52). These results further support the potential association between miRNA clusters and immunity, and even the mechanism of MG. Additionally, 10 miRNA clusters that have not been reported previously were identified through the global analysis of MG risk miRNA clusters in the present study. These novel identified clusters may provide novel insight into future experimental studies of miRNA clusters in MG and immune-associated diseases.

In conclusion, the present study created a catalog of MG risk genes and miRNAs, acquired MG risk pathways, located each MG risk miRNA on each chromosome, obtained 15 significant miRNA clusters associated with MG, and identified the risk pathways of each of the 15 miRNA clusters. This involved the integration of a number of single miRNA studies into a global study of miRNA clusters in MG. As the number of studies on high-throughput data continues to increase, the results of the present study provide supporting evidence and offer novel insight for further investigations on miRNA clusters in the pathogenesis of MG.

\section{Acknowledgements}

Not applicable.

\section{Funding}

The present study was supported by the National Natural Science Foundation of China (grant nos. 81571166,81771361, 81701190 and 81820108014), the Applied Technique Research and Development Project of Harbin (grant no. 2016RAXYJ067), the Health and Family Planning Commission of Heilongjiang Province (grant nos. 2016-052 and 2016-072) and the Fundamental Research Funds for the Provincial Universities (grant nos. 2017LCZX57, 2017LCZX65 and 2017LCZX48).

\section{Availability of data and materials}

All data generated and/or analyzed during the present study are included in this published article.

\section{Authors' contributions}

LW, SN, JW, CB and HZ designed the study. TW, YW and SL collected the data. CB, HZ, YC, XL, XK, XS and ZL were involved in the interpretation of data. $\mathrm{CB}$ and JW analyzed and visualized the data. CB drafted the manuscript. SN and 
LW revised the manuscript. All authors read and approved the final manuscript.

\section{Ethics approval and consent to participate}

Not applicable.

\section{Patient consent for publication}

Not applicable.

\section{Competing interests}

The authors declare that they have no competing interests.

\section{References}

1. Conti-Fine BM, Milani M and Kaminski HJ: Myasthenia gravis: Past, present and future. J Clin Invest 116: 2843-2854, 2006.

2. Cavalcante P, Barberis M, Cannone M, Baggi F, Antozzi C, Maggi L, Cornelio F, Barbi M, Dido P, Berrih-Aknin S, et al: Detection of poliovirus-infected macrophages in thymus of patients with myasthenia gravis. Neurology 74: 1118-1126, 2010.

3. Xie Y, Li HF, Jiang B, Li Y, Kaminski HJ and Kusner LL: Elevated plasma interleukin-17A in a subgroup of Myasthenia Gravis patients. Cytokine 78: 44-46, 2016.

4. Lee JS, Joo IS and Seok JI: Widely varying TNF-alpha levels in patients with myasthenia gravis. Neurol Sci 30: 259-262, 2009.

5. Li Q, Liu P, Xuan X, Zhang J, Zhang Y, Zhu Z, Gao F, Zhang Q and Du Y: CCR9 AND CCR7 are overexpressed in CD4 CD8- thymocytes of myasthenia gravis patients. Muscle Nerve 55: 84-90, 2017.

6. Yang L, Wang J, Sun X, Cao Y, Ning S, Zhang H, Chen L, Li R, Tian Q, Wang L, et al: Identifying a polymorphic 'switch' that influences miRNAs' regulation of a myasthenia gravis risk pathway. PLoS One 9: e104827, 2014.

7. Cao Y, Lu X, Wang J, Zhang H, Liu Z, Xu S, Wang T, Ning S, Xiao B and Wang L: Construction of an miRNA-regulated drug-pathway network reveals drug repurposing candidates for myasthenia gravis. Int J Mol Med 39: 268-278, 2017.

8. Xin Y, Cai H, Lu T, Zhang Y, Yang Y and Cui Y: miR-20b Inhibits $T$ cell proliferation and activation via NFAT signaling pathway in thymoma-associated myasthenia gravis. Biomed Res Int 2016: 9595718, 2016.

9. Li J, Qiu D, Chen Z, Du W, Liu J and Mo X: Altered expression of miR-125a-5p in thymoma-associated myasthenia gravis and its down-regulation of foxp3 expression in Jurkat cells. Immunol Lett 172: 47-55, 2016

10. Liu XF, Wang RQ, Hu B, Luo MC, Zeng QM, Zhou H, Huang K, Dong XH, Luo YB, Luo ZH and Yang H: MiR-15a contributes abnormal immune response in myasthenia gravis by targeting CXCL10. Clin Immunol 164: 106-113, 2016.

11. Leonardo TR, Schultheisz HL, Loring JF and Laurent LC: The functions of microRNAs in pluripotency and reprogramming. Nat Cell Biol 14: 1114-1121, 2012.

12. Kästle M, Bartel S, Geillinger-Kästle K, Irmler M, Beckers J, Ryffel B, Eickelberg O and Krauss-Etschmann S: microRNA cluster 106a 363 is involved in T helper 17 cell differentiation. Immunology 152: 402-413, 2017.

13. Kuppers DA, Schmitt TM, Hwang HC, Samraj L, Clurman BE and Fero ML: The miR-106a 363 ${ }^{\text {xpl1 }}$ miRNA cluster induces murine T cell lymphoma despite transcriptional activation of the p2 $7^{\text {Kipl }}$ cell cycle inhibitor. Oncotarget 8: 50680-50691, 2017.

14. Kurkewich JL, Hansen J, Klopfenstein N, Zhang H, Wood C, Boucher A, Hickman J, Muench DE, Grimes HL and Dahl R: The miR-23a 27a 24-2 microRNA cluster buffers transcription and signaling pathways during hematopoiesis. PLoS Genet 13: e1006887, 2017.

15. Pinero J, Queralt-Rosinach N,Bravo A, Deu-Pons J, Bauer-Mehren A, Baron M, Sanz F and Furlong LI: DisGeNET: A discovery platform for the dynamical exploration of human diseases and their genes. Database (Oxford) 2015: bav028, 2015.

16. Amberger JS, Bocchini CA, Schiettecatte F, Scott AF and Hamosh A: OMIM.org: Online mendelian inheritance in man (OMIM $\left.{ }^{\circledR}\right)$, an online catalog of human genes and genetic disorders. Nucleic Acids Res 43 (Database Issue): D789-D798, 2015.
17. Kozomara A and Griffiths-Jones S: miRBase: Integrating microRNA annotation and deep-sequencing data. Nucleic Acids Res 39 (Database Issue): D152-D157, 2011.

18. Brown GR, Hem V, Katz KS, Ovetsky M, Wallin C, Ermolaeva O, Tolstoy I, Tatusova T, Pruitt KD, Maglott DR and Murphy TD: Gene: A gene-centered information resource at NCBI. Nucleic Acids Res 43 (Database Issue): D36-D42, 2015.

19. Wang J, Cao Y, Zhang H, Wang T, Tian Q, Lu X, Lu X, Kong X, Liu Z, Wang N, et al: NSDNA: A manually curated database of experimentally supported ncRNAs associated with nervous system diseases. Nucleic Acids Res 45: D902-D907, 2017.

20. Hsu SD, Tseng YT, Shrestha S, Lin YL, Khaleel A, Chou CH, Chu CF, Huang HY, Lin CM, Ho SY, et al: miRTarBase update 2014: An information resource for experimentally validated miRNA-target interactions. Nucleic Acids Res 42 (Database Issue): D78-D85, 2014.

21. Kanehisa M and Goto S: KEGG: Kyoto encyclopedia of genes and genomes. Nucleic Acids Res 28: 27-30, 2000.

22. Dennis G Jr, Sherman BT, Hosack DA, Yang J, Gao W, Lane HC and Lempicki RA: DAVID: Database for annotation, visualization and integrated discovery. Genome Biol 4: P3, 2003.

23. Ashburner M, Ball CA, Blake JA, Botstein D, Butler H, Cherry JM, Davis AP, Dolinski K, Dwight SS, Eppig JT, et al: Gene ontology: Tool for the unification of biology. The gene ontology consortium. Nat Genet 25: 25-29, 2000.

24. Smith-Bouvier DL, Divekar AA, Sasidhar M, Du S, Tiwari-Woodruff SK, King JK, Arnold AP, Singh RR and Voskuhl RR: A role for sex chromosome complement in the female bias in autoimmune disease. J Exp Med 205: 1099-1108, 2008.

25. Chitnis T and Khoury SJ: Sex influences in autoimmune disease. Clin Immunol 149: 169, 2013

26. Zhu ML, Bakhru P, Conley B, Nelson JS, Free M, Martin A, Starmer J, Wilson EM and Su MA: Sex bias in CNS autoimmune disease mediated by androgen control of autoimmune regulator. Nat Commun 7: 11350, 2016.

27. Cao M, Seike M, Soeno C, Mizutani H, Kitamura K, Minegishi Y, Noro R, Yoshimura A, Cai L and Gemma A: MiR-23a regulates TGF- $\beta$-induced epithelial-mesenchymal transition by targeting E-cadherin in lung cancer cells. Int J Oncol 41: 869-875, 2012.

28. Kitamura K, Seike M, Okano T, Matsuda K, Miyanaga A, Mizutani H, Noro R, Minegishi Y, Kubota K and Gemma A: MiR-134/487b/655 cluster regulates TGF- $\beta$-induced epithelial-mesenchymal transition and drug resistance to gefitinib by targeting MAGI2 in lung adenocarcinoma cells. Mol Cancer Ther 13: 444-453, 2014

29. Suffert G, Malterer G, Hausser J, Viiliainen J, Fender A, Contrant M, Ivacevic T, Benes V, Gros F, Voinnet O, et al: Kaposi's sarcoma herpesvirus microRNAs target caspase 3 and regulate apoptosis. PLoS Pathog 7: e1002405, 2011.

30. Naidu S, Shi L, Magee P, Middleton JD, Lagana A, Sahoo S, Leong HS, Galvin M, Frese K, Dive C, et al: PDGFR-modulated miR-23b cluster and miR-125a-5p suppress lung tumorigenesis by targeting multiple components of KRAS and NF-kB pathways. Sci Rep 7: 15441, 2017.

31. Li SG, Shi QW, Yuan LY, Qin LP, Wang Y, Miao YQ, Chen Z, Ling CQ and Qin WX: C-Myc-dependent repression of two oncogenic miRNA clusters contributes to triptolide-induced cell death in hepatocellular carcinoma cells. J Exp Clin Cancer Res 37: 51, 2018 .

32. Llobet-Navas D, Rodriguez-Barrueco R, Castro V, Ugalde AP, Sumazin P, Jacob-Sendler D, Demircan B, Castillo-Martin M, Putcha P, Marshall N, et al: The miR-424 (322)/503 cluster orchestrates remodeling of the epithelium in the involuting mammary gland. Genes Dev 28: 765-782, 2014.

33. Cavalcante P, Galbardi B, Franzi S, Marcuzzo S, Barzago C, Bonanno S, Camera G, Maggi L, Kapetis D, Andreetta F, et al: Increased expression of Toll-like receptors 7 and 9 in myasthenia gravis thymus characterized by active Epstein-Barr virus infection. Immunobiology 221: 516-527, 2016.

34. Grauer O, Wolff D, Bertz H, Greinix H, Kuhl JS, Lawitschka A, Lee SJ, Pavletic SZ, Holler E and Kleiter I: Neurological manifestations of chronic graft-versus-host disease after allogeneic haematopoietic stem cell transplantation: Report from the Consensus Conference on Clinical Practice in chronic graft-versus-host disease. Brain 133: 2852-2865, 2010.

35. Unal S, Sag E, Kuskonmaz B, Kesici S, Bayrakci B, Ayvaz DC, Tezcan I, Yalnizoglu D and Uckan D: Successful treatment of severe myasthenia gravis developed after allogeneic hematopoietic stem cell transplantation with plasma exchange and rituximab. Pediatr Blood Cancer 61: 928-930, 2014. 
36. Heidarzadeh Z, Mousavi SA, Ostovan VR and Nafissi S: Muscle-specific kinase antibody associated myasthenia gravis after bone marrow transplantation. Neuromuscul Disord 24 148-150, 2014.

37. Cioffi M, Trabulo SM, Vallespinos M, Raj D, Kheir TB, Lin ML, Begum J, Baker AM, Amgheib A, Saif J, et al: The miR-25-93-106b cluster regulates tumor metastasis and immune evasion via modulation of CXCL12 and PD-L1. Oncotarget 8: 21609-21625, 2017.

38. Punga T, Bartoccioni E, Lewandowska M, Damato V, Evoli A and Punga AR: Disease specific enrichment of circulating let-7 family microRNA in MuSK+ myasthenia gravis. J Neuroimmunol 292: 21-26, 2016.

39. Wu Y, Heinrichs J, Bastian D, Fu J, Nguyen H, Schutt S, Liu Y, Jin J, Liu C, Li QJ, et al: MicroRNA-17-92 controls T-cell responses in graft-versus-host disease and leukemia relapse in mice. Blood 126: 1314-1323, 2015.

40. Wu D, Bi X, Qu L, Han L, Yin C, Deng J, Dong Z, Mi QS and Zhou L: miRNA miR-17-92 cluster is differentially regulated in the imiqumod-treated skin but is not required for imiqumod-induced psoriasis-like dermatitis in mice. Exp Dermatol 26: 82-84, 2017.

41. de Kouchkovsky D, Esensten JH, Rosenthal WL, Morar MM Bluestone JA and Jeker LT: microRNA-17-92 regulates IL-10 production by regulatory $\mathrm{T}$ cells and control of experimental autoimmune encephalomyelitis. J Immunol 191: 1594-1605, 2013.

42. Geng XR, Qiu SQ, Yang LT, Liu ZQ, Yang G, Liu JQ, Zeng L, Li XX, Mo LH, Liu ZG and Yang PC: Allergen-specific immune response suppresses interleukin 10 expression in $\mathrm{B}$ cells via increasing micro-RNA-17-92 cluster. Cell Biochem Funct 34 449-454, 2016

43. Chandran PA, Keller A, Weinmann L, Seida AA, Braun M, Andreev K, Fischer B, Horn E, Schwinn S, Junker M, et al: The TGF- $\beta$-inducible miR-23a cluster attenuates IFN- $\gamma$ levels and antigen-specific cytotoxicity in human $\mathrm{CD}^{+} \mathrm{T}$ cells. J Leukoc Biol 96: 633-645, 2014
44. Cho S, Wu CJ, Yasuda T, Cruz LO, Khan AA, Lin LL, Nguyen DT, Miller M, Lee HM, Kuo ML, et al: miR-23 approximately 27 approximately 24 clusters control effector $\mathrm{T}$ cell differentiation and function. J Exp Med 213: 235-249, 2016.

45. Kurkewich JL, Bikorimana E, Nguyen T, Klopfenstein N, Zhang H, Hallas WM, Stayback G, McDowell MA and Dahl R: The mirn23a microRNA cluster antagonizes B cell development. J Leukoc Biol 100: 665-677, 2016.

46. Ventura A, Young AG, Winslow MM, Lintault L, Meissner A, Erkeland SJ, Newman J, Bronson RT, Crowley D, Stone JR, et al: Targeted deletion reveals essential and overlapping functions of the miR-17 through 92 family of miRNA clusters. Cell 132: 875-886, 2008.

47. Jiang S, Li C, Olive V, Lykken E, Feng F, Sevilla J, Wan Y, He L and Li QJ: Molecular dissection of the miR-17-92 cluster's critical dual roles in promoting Th1 responses and preventing inducible Treg differentiation. Blood 118: 5487-5497, 2011

48. Khan AA, Penny LA, Yuzefpolskiy Y, Sarkar S and Kalia V: MicroRNA-17 92 regulates effector and memory CD8 T-cell fates by modulating proliferation in response to infections. Blood 121: 4473-4483, 2013.

49. Olive V, Li Q and He L: mir-17-92: A polycistronic oncomir with pleiotropic functions. Immunol Rev 253: 158-166, 2013.

50. Wang B, Zhang C, Zhang A, Cai H, Price SR and Wang XH MicroRNA-23a and MicroRNA-27a mimic exercise by ameliorating CKD-induced muscle atrophy. J Am Soc Nephrol 28: 2631-2640, 2017

51. Su R, Dong L, Zou D, Zhao H, Ren Y, Li F, Yi P, Li L, Zhu Y, Ma Y, et al: microRNA-23a, -27a and -24 synergistically regulate JAK1/Stat 3 cascade and serve as novel therapeutic targets in human acute erythroid leukemia. Oncogene 35: 6001-6014, 2016.

52. Li X, Liu X, Xu W, Zhou P, Gao P, Jiang S, Lobie PE and Zhu T: c-MYC-regulated miR-23a/24-2/27a cluster promotes mammary carcinoma cell invasion and hepatic metastasis by targeting Sprouty2. J Biol Chem 288: 18121-18133, 2013. 